\title{
DINÁMICA ACTUAL DE LOS NICHOS DE NIVACIÓN DEL ALTO SIL (CORDILLERA CANTÁBRICA)
}

\author{
J. SANTOS GONZÁLEZ, J. M. REDONDO VEGA, \\ A. GÓMEZ VILLAR y R. B. GONZÁLEZ GUTIÉRREZ
}

\author{
Departamento de Geografía y Geología. \\ Universidad de León. Campus de Vegazana, s/n. 24071 León \\ Correo electrónico de contacto: jsango@unileon.es
}

\begin{abstract}
RESUMEN: Entre los años 2003 y 2009 se ha realizado un seguimiento de los neveros más persistentes de un sector de la Cordillera Cantábrica, el Alto Sil. Este trabajo ha permitido localizar 8 nichos de nivación dentro de este territorio. Seis de ellos se pueden considerar activos actualmente y 2 inactivos. El estudio más detallado de dos enclaves, nichos de las Joyas del Nevadín y Valdeiglesia, ha permitido comprobar la actividad erosiva de la nieve, observándose una notable relación entre la cantidad de nieve en primavera y la dinámica nival. El lento deslizamiento de la nieve ladera abajo se traduce en la aparición de estrías de nevero y en el arrastre de pequeños cantos. Además, el desgaste de los afloramientos rocosos es intenso en estos lugares. Por otra parte, se ha analizado la duración de la cubierta nival actualmente en estos enclaves, que parece haberse reducido de forma notable en los últimos 20-30 años, probablemente tanto por una disminución del volumen total de nieve que reciben estas montañas, como por un cambio de usos del suelo por la reducción de la carga ganadera que soportaban.
\end{abstract}

ABSTRACT:From 2003 to 2009 it has been tracked snowpatches in Alto Sil, in the Cantabrian Mountains. This work allowed us to locate 8 nivation hollows in this area. Currently 6 of them could be considered as active and 2 of them as inactive. A more detail study around Joyas del Nevadín and Valdeiglesia nivation hollows, allowed us to check the snow erosion activity. It was observed a strong relation between the quantity of snow in spring and the dinamic of the nivation hollow. The snow sliding provokes nival striae and clast displacements. Besides, the erosion of rock outcrops is intense in this area. In these locations, it has also been analyzed the duration of the snow cover which seems to be reduced in the last 20-30 years. This can be due to a decrease in the snow volume in these mountains and to changes on land uses.

Palabras clave: Cordillera Cantábrica, nichos de nivación, acción nival.

Key words: Cantabrian Mountains, nivation hollows, snow action. 


\section{Introducción}

Los neveros constituyen zonas relativamente pequeñas donde la cubierta nival permanece después del período general de fusión, siendo normalmente remanentes de bancos de nieve acumulados por el viento (snowdrifts) (van Everdingen, 2005). Pueden ser temporales, si funden en algún momento del año, o permanentes, si mantienen nieve durante todo el año.

El papel erosivo de la nieve ha sido muy discutido. Sin embargo, diversos estudios demuestran que el lento deslizamiento de la nieve puede generar formas muy similares a las de un glaciar, desarrollándose tanto formas erosivas como acumulativas significativas (Matthes, 1911; Costin et al., 1973; Jennings y Costin, 1978; Shakesby, 1997; Shakesby et al., 1999; Carrera Gómez et al., 2006).

En cualquier caso, para que la nieva ejerza un papel erosivo, es necesario que ésta se desplace, para lo que se necesitan un espesor de nieve y una pendiente mínimos, además de que la nieve se densifique notablemente. En este sentido, Bowman (1916), a partir de neveros en clima tropical, establece una curva que relaciona el espesor mínimo de nieve necesario para que ésta se desplace y la pendiente sobre la que se asienta. Según este autor, con pendientes de $15^{\circ}$ se requieren entre 15 y $30 \mathrm{~m}$ de nieve, mientras que si la pendiente alcanza los $20^{\circ}$ el movimiento puede producirse entre 7 y $15 \mathrm{~m}$ de nieve, valores que se pueden alcanzar en determinados enclaves del noroeste peninsular, como ocurre en el Alto Sil.

En ocasiones, la erosión provocada por la existencia de estos neveros puede generar una forma semi-circular, muy similar a un circo glaciar de pequeño tamaño, que se denomina nicho de nivación o nicho nival (Lewis, 1939). Tradicionalmente se ha considerado que su crecimiento se debía a la erosión por gelifracción en el contacto entre la parte superior del nevero y la ladera (Gardner, 1969), pero el mecanismo de crecimiento de los nichos de nivación es aún muy mal conocido (Nyberg, 1991; Thorn y Hall, 2002). En realidad, no existe un criterio definitivo para la distinción entre circos glaciares y nichos nivales (Nelson y Jackson, 2003) puesto que, aunque suelen ser más pequeños, no pueden ser diferenciados sólo por eso (Evans y Cox, 1974). De cualquier forma, su grado de desarrollo es muy superior sobre rocas blandas que sobre sustrato resistente (Christiansen, 1998). En algunos casos, los nichos de nivación parecen evolucionar a partir de la acumulación de nieve en deslizamientos en masa (Palacios Estremera et al., 2004), aunque no siempre ocurre así, siendo más habitual que se generen en la parte superior de antiguos circos glaciares.

En la Cordillera Cantábrica y en otras zonas del noroeste peninsular existen algunos nichos de nivación, habitualmente orientados al NE o E, donde la nieve, aún hoy en día, puede permanecer hasta bien entrado el verano pero, al igual que ocurre en el Pirineo (Serrano et al., 2000), son citados sólo en contadas ocasiones (González Gutiérrez, 2002) y, únicamente en trabajos recientes se estudia la dinámica del nevero del Cuiña, en la Sierra de Ancares (Valcárcel Díaz et al., 2005; Carrera Gómez et al., 2006). Más atención han recibido algunos neveros existentes en el Sistema Central (Palacios Estremera y García Sánchez-Colomer, 1997; Palacios Estremera et al., 2002; 2003; 2004). 


\section{2. Área de estudio}

El Alto Sil se encuentra en la vertiente meridional del sector occidental de la Cordillera Cantábrica, en el noroeste de la provincia de León. Se trata de un territorio organizado por el río Sil y sus afluentes, con cotas comprendidas entre los 1.900-2.100 m de las cumbres y los 750-900 m del fondo de valle principal (Fig. 1).

El clima es oceánico, con precipitaciones que oscilan entre los $1.000 \mathrm{~mm}$ anuales del extremo SW y los más de $2.000 \mathrm{~mm}$ que reciben las zonas más elevadas. Buena parte de las precipitaciones caen en forma de nieve, siendo ésta abundante por encima de los $1.600 \mathrm{~m}$ entre diciembre y abril, existiendo pequeños enclaves en los que está presente entre noviembre y comienzos del verano de manera habitual.

Los materiales que integran el sustrato del Alto Sil son del Precámbrico y del Paleozoico Inferior, predominando los materiales silíceos (cuarcitas, pizarras y, en menor medida, areniscas), existiendo sólo pequeños afloramientos calcáreos.

Toda la zona presenta abundantes huellas morfológicas de la acción glaciar cuaternaria, especialmente en los tramos de valle con orientación N-S o E-W, donde las for-

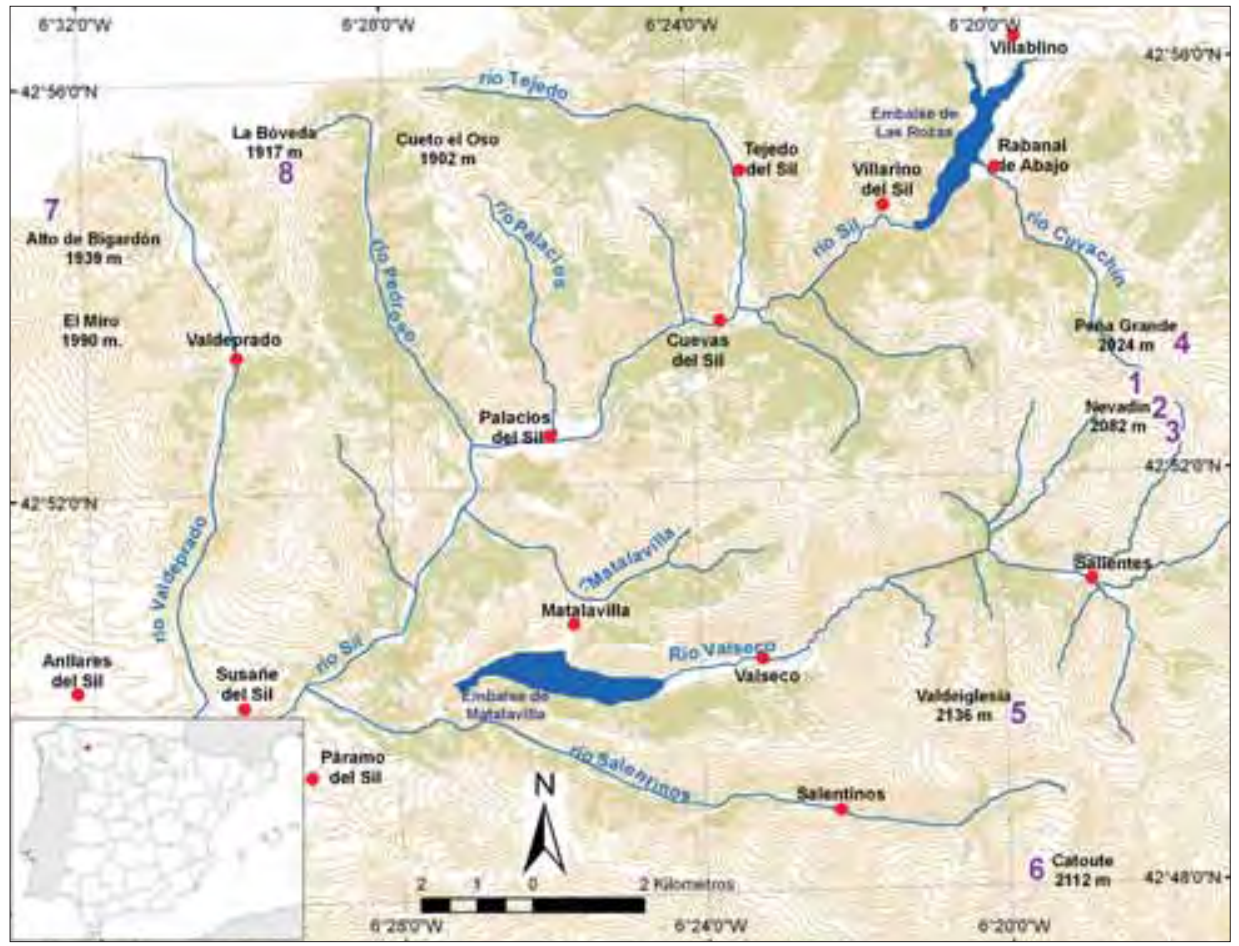

Figura 1. Localización del área de estudio. Los números indican los nichos de nivación del Alto Sil, correspondiéndose con los que aparecen en la Tabla 1. 
mas son más rotundas (Redondo Vega, 2002). Durante el máximo glaciar, anterior al 35.000 A.P. (Jalut et al., 2004), se desarrolló un gran complejo glaciar, probablemente el mayor de toda la Cordillera Cantábrica, que dejó restos evidentes en todos los valles (Redondo Vega, 2002; Redondo Vega et al., 2006; García de Celis y Martínez Fernández, 2002).

Destaca, especialmente, el conjunto de bloques erráticos (Santos González et al., 2006) y depósitos glacio-lacustres, glaciares y fluvio-glaciares de los entornos de Páramo y Susañe del Sil, a cotas de 760-900 m, declarados, recientemente, como Lugares de Interés Geológico (Fernández Martínez y Fuertes Gutiérrez, 2009). Tras la deglaciación, se desarrollaron numerosos glaciares rocosos perfectamente conservados y que constituyen un conjunto muy singular en el contexto peninsular (Redondo Vega et al., 2004; Fernández Martínez y Fuertes Gutiérrez, 2009).

\section{Metodología}

Entre los años 2003 y 2009 se ha analizado la dinámica de los neveros de las Joyas del Nevadín y Valdeiglesia, situados en la Sierra de Gistredo. Además, se ha realizado un seguimiento más superficial de los neveros más persistentes del resto del Alto Sil. Todo ello ha permitido definir los lugares con mayor persistencia nival en todo este territorio.

Para observar la dinámica nival, en otoño de 2003 se instalaron una serie de varillas de hierro en los neveros de las Joyas del Nevadín y Valdeiglesia, los dos enclaves con mayor persistencia de nieve de todo el Alto Sil. Se trata de varillas de hierro de 1,5 $\mathrm{m}$ de longitud, que fueron insertadas verticales $1 \mathrm{~m}$ en el suelo, si bien en algunos casos el espesor de suelo hizo que no se pudiera profundizar más que $20-30 \mathrm{~cm}$. En el nevero del Nevadín se colocaron un total de 16 varillas en tres filas, transversales a la pendiente de la ladera. En el nevero de Valdeiglesia se instalaron del mismo modo un total de 15 varillas.

En ambos lugares, a primeros de noviembre de 2004, se trazaron líneas de pintura con el fin de evaluar la dinámica actual dentro de los neveros y el arrastre de materiales. Las líneas de pintura fueron realizadas con trazador de obras SETTOP, un aerosol ecológico de pintura fluorescente de marcación, de color azul y secado rápido. Para su trazado se siguió una línea de referencia con el objetivo de pintar líneas lo más rectas posible. Las líneas atravesaban zonas tanto de afloramientos de roquedo de pizarras, como de materiales sueltos, con el fin de comparar los posibles movimientos en ambas zonas.

Los observaciones sobre la inclinación de las varillas y sobre la evolución de las líneas de pintura se hicieron todos los años tanto poco después de la fusión de la nieve como en otoño, antes del establecimiento de la cubierta nival, con el fin de diferenciar los desplazamientos producidos en invierno de los que pudieran darse en los meses estivales, ya que las parcelas analizadas no fueron valladas y, al menos en el caso del Nevadín, existe un uso ganadero del entorno en los meses estivales. 


\section{Resultados}

\subsection{Distribución de los nichos de nivación en el Alto Sil}

En el Alto Sil hemos localizado ocho nichos de nivación (Tabla 1), en el entorno de las cumbres de Valdeiglesia (2.136 m), Catoute (2.112 m), Nevadín (2.077 m), Peña Grande (2025 m), Alto de Bigardón (1.939 m) y La Bóveda (1.917 m). De ellos, seis pueden considerarse activos, puesto que presentan zonas desprovistas casi totalmente de vegetación y desplazamientos de cantos recientes, destacando sobre todos los demás los de Valdeiglesia y, en especial, las Joyas del Nevadín, donde la persistencia de la nieve y la dinámica nival es mucho más activa que en el resto.

Tabla 1. Nichos de nivación en el Alto Sil. La superficie dinámica se refiere a zonas donde no hay vegetación y la cubierta liquénica es muy escasa, observándose procesos erosivos evidentes.

\begin{tabular}{|c|c|c|c|c|c|c|c|}
\hline $\mathbf{N}^{0}$ & Denominación & Orientación & $\begin{array}{l}\text { Altitud } \\
\text { (m.sn.m) }\end{array}$ & $\begin{array}{c}\text { Anchura } \\
\text { (m) }\end{array}$ & $\begin{array}{l}\text { Longitud } \\
\text { (m) }\end{array}$ & $\begin{array}{l}\text { Superficie } \\
\text { Total }\left(\mathbf{m}^{2}\right)\end{array}$ & $\begin{array}{c}\text { Superficie } \\
\text { dinámica }\left(\mathbf{m}^{2}\right)\end{array}$ \\
\hline 1 & Nevadín Norte & $\mathrm{NE}$ & 2072-1992 & 167 & 38 & 6396 & 1546 \\
\hline 2 & Joyas del Nevadín & E-NE & $2060-2010$ & 148 & 54 & 5370 & 3002 \\
\hline 3 & Joyas del Nevadín S & $\mathrm{NE}$ & 2028-2000 & 121 & 34 & 2954 & 1901 \\
\hline 4 & Peña Grande & E & 2012-1988 & 100 & 39 & 3601 & 349 \\
\hline 5 & Valdeiglesia & $\mathrm{NE}$ & $2040-2000$ & 190 & 57 & 7009 & 2364 \\
\hline 6 & Los Fueyos & NE & 2030-2005 & 96 & 26 & 1810 & 1810 \\
\hline 7 & Fasgueo & $\mathrm{N}$ & $1912-1900$ & 62 & 21 & 1044 & Inactivo \\
\hline 8 & La Bóveda & $S$ & $1905-1895$ & 60 & 18 & 971 & Inactivo \\
\hline \multicolumn{3}{|c|}{ MEDIA } & 2007-1974 & 118 & 36 & 3644 & 1372 \\
\hline
\end{tabular}

Los nichos actualmente activos se encuentran en torno a los $2.000 \mathrm{~m}$ de altitud y se sitúan en zonas de sotavento, en el borde superior de algunos circos glaciares. Son formas más anchas que largas, semi-circulares y con pendientes de en torno a $30^{\circ}$ en la parte superior y $20-22^{\circ}$ en la zona inferior (Fig. 2).

El tamaño de estos nichos de nivación puede alcanzar las 0,7 hectáreas, siendo más pequeños que los que aparecen en otras zonas de la Cordillera Cantábrica (González Gutiérrez, 2002) y los que hemos observado en otros macizos del noroeste peninsular, como la Sierra Segundera o los Montes Aquilanos. Aunque están mejor desarrollados sobre las pizarras precámbricas (áreas de Nevadín y Peña Grande), lo cierto es que hay algunos ejemplos labrados en las cuarcitas de la Serie de los Cabos (Valdeiglesia y Los Fueyos), si bien presentan formas más alargadas y menos profundas, debido a la mayor resistencia del sustrato. 


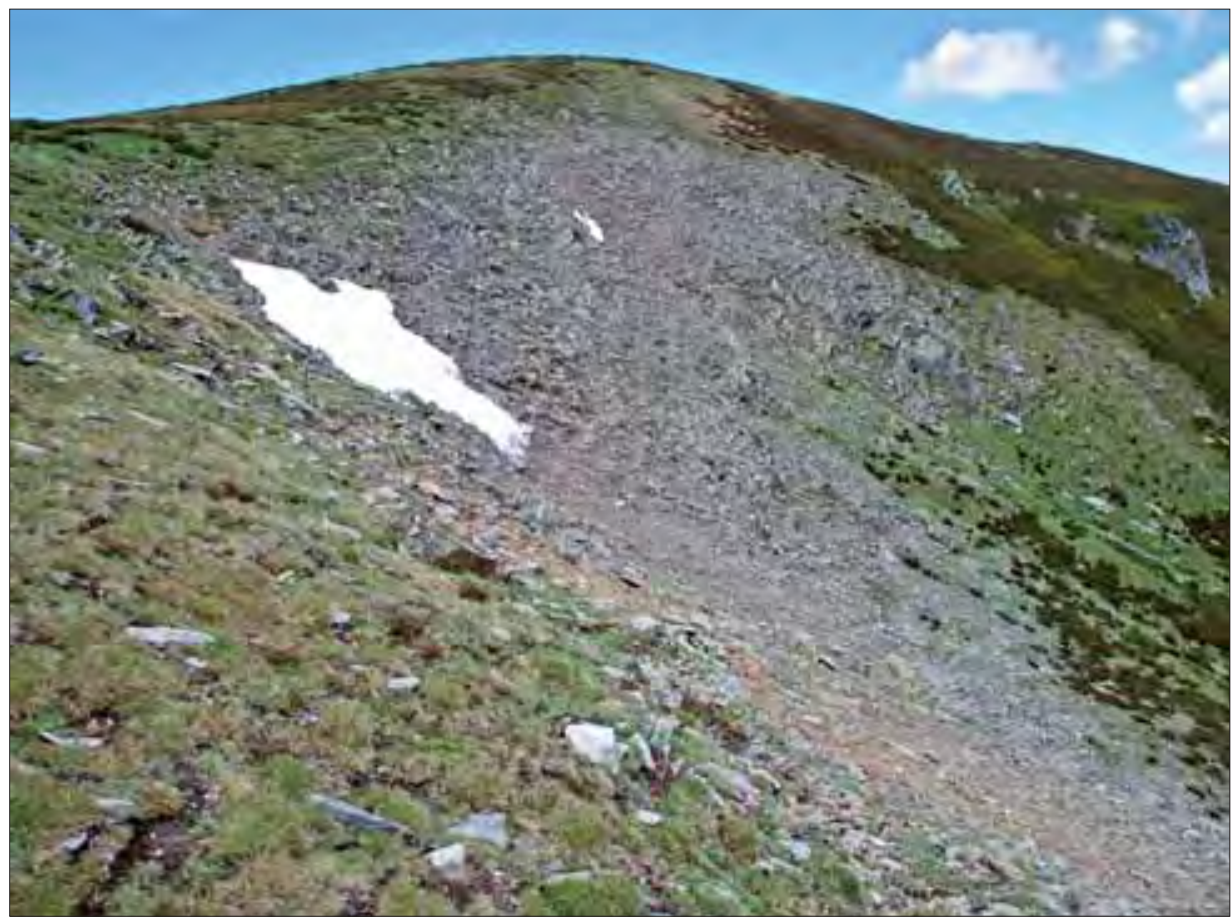

Figura 2. Nicho de nivación de las Joyas del Nevadín. Destaca la casi total ausencia de vegetación en su interior, contrastando con las aéreas cercanas, colonizadas por vegetación herbácea y arbustiva.

Además de estos nichos de nivación, con frecuencia perviven durante largo tiempo, llegando incluso a comienzos del verano, algunas manchas de nieve en otras ubicaciones donde la acción de la nieve tiene una menor influencia y no se crean los citados nichos. Esto ocurre por ejemplo a los pies de la Peña Carnicera (Salientes), a 1.950 m, o en la parte superior del fondo del circo de Los Fueyos (Salentinos), a $2.030 \mathrm{~m}$.

La acumulación y persistencia de la nieve en estos enclaves depende de factores muy locales y no tiene por qué coincidir con los años de densa cubierta nival en el resto del Alto Sil. En realidad, se trata de sistemas caracterizados por la existencia de una topografía plana a barlovento, muy expuesta a los vientos del SW (del $\mathrm{N}$ en el caso de La Bóveda), con una cubierta arbustiva escasa o nula y, adyacente a ella, una zona muy favorable a la acumulación nival, donde se produce un brusco cambio de pendiente, en el borde superior de los circos glaciares (Fig. 3).

Esta zona recibe la nieve caída en el área de barlovento, siempre y cuando la dirección de los vientos sea la adecuada. En estas zonas, como suele ocurrir en las áreas cimeras de las zonas montañosas, el transporte de la nieve por el viento es un mecanismo muy 


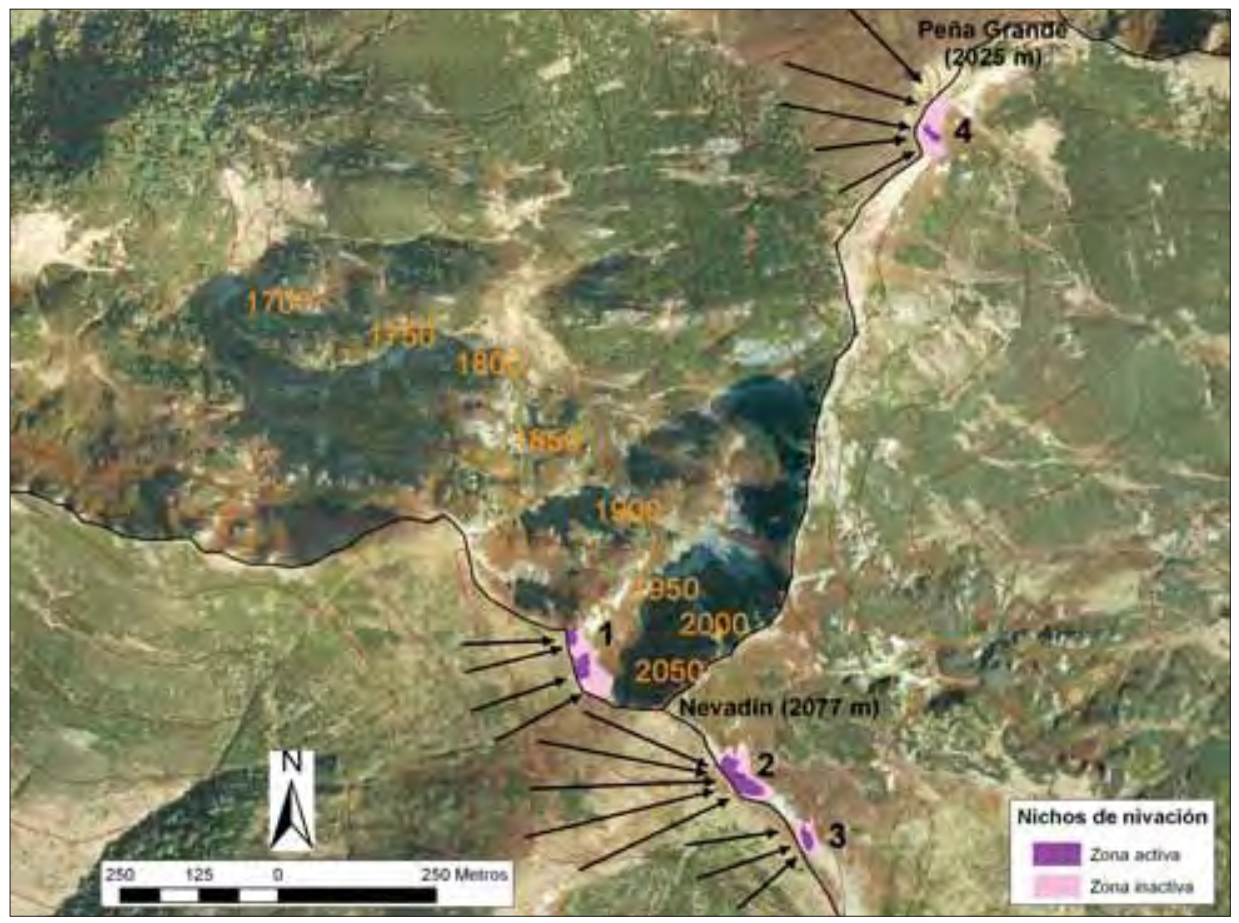

Figura 3. Situación de los nichos de nivación en el entorno de los picos Nevadín y Peña Grande. En las denominadas zonas activas se observan arrastres y estrías de nevero, mientras que las inactivas aparecen colonizadas por vegetación. Las flechas negras indican las direcciones de viento predominantes responsables de la acumulación de nieve en los diferentes nichos nivales. Ortofotografía: Junta de Castilla y León, Año 2008.

eficaz, pudiéndose acumular volúmenes de nieve muy superiores a los que realmente precipitan (Mases et al., 1998; Corripio et al., 2004; David, 2004; Guyomarc'h et al., 2004).

Existe una orientación preferente al NE, pues se conjugan, por una parte, la mejor conservación de la nieve y, por otra, la mayor sobre-acumulación de nieve, puesto que ésta procede mayoritariamente de componente SW o W. Por eso, es aquí donde se localizan los neveros más persistentes. Por tanto, las topografías suaves orientadas al SW y la presencia de circos glaciares hacia el NE influyen mucho en la mayor acumulación de nieve en esas zonas, al igual que sucede en otras muchas montañas del Hemisferio Norte (Palacios Estremera et al., 2002; Watson et al., 1994). Este sistema aparece en el borde superior de todos los circos glaciares, sólo que en algunos es mucho más eficaz que en otros, bien por una mayor superficie a barlovento, por una topografía que favorece más la acumulación de nieve en un enclave concreto o por una menor insolación. Si, además, las condiciones de pendiente y sustrato son las adecuadas, se generan nichos de nivación. 


\subsection{Duración de la nieve en los nichos de nivación}

El establecimiento definitivo de la nieve en los nichos de nivación no se suele producir con las primeras nevadas, que habitualmente se dan a primeros del mes de octubre (ocasionalmente antes, incluso en pleno verano, como en agosto de 2006), sino con nevadas posteriores, a finales de ese mes o de primeros de noviembre. Una vez instalada la cubierta nival y, a diferencia de otros muchos sectores de la sierra, ésta no desaparece (salvo condiciones excepcionales, como en enero de 2007), hasta finales de primavera.

El espesor de nieve va aumentando con las nevadas normalmente hasta mediados de marzo, aunque se pueden producir precipitaciones en forma de lluvia en cualquier mes. Posteriormente, hay tanto fusión como acumulación, gracias a nuevas nevadas que suelen tener menor entidad, aunque en abril son relativamente frecuentes las nevadas e incluso pueden ser importantes aún en mayo. A finales de abril del 2006 pudimos observar grandes espesores de nieve, que superaban los $5 \mathrm{~m}$ en neveros situados unos $100 \mathrm{~m}$ más bajos que el del Nevadín, estimándose un espesor superior a los $8 \mathrm{~m}$ en el nevero monitoreado.

Esta nieve se va densificando y transformando notablemente, humedeciéndose sensiblemente durante la primavera, con períodos de hielo/deshielo de la superficie muy frecuentes. Si el espesor de nieve es el suficiente (probablemente en torno a los 6-8 m), esa nieve se desplaza lentamente ladera abajo. Ese movimiento de la nieve queda marcado por la presencia de grietas en las cornisas superiores de los neveros, que indican el movimiento de ésta por efecto de la gravedad hacia zonas inferiores (Fig. 4). En el nevero de las Joyas del Nevadín, hemos observado como las grietas de tracción cambian cada año de posición, dependiendo del volumen total de nieve acumulado, aspecto señalado también en neveros de Groenlandia (Christiansen, 1998) y Japón (Daimaru et al., 2002).

Después de ese período en el que, siempre y cuando el espesor de nieve haya sido el suficiente, el nevero permanece activo, la nieve va desapareciendo rápidamente con las altas temperaturas de finales de primavera y comienzos del verano. Esta fusión, en los años de seguimiento que llevamos hasta ahora, ha sido muy variable, pudiendo variar en casi dos meses según las condiciones meteorológicas de cada año (Tabla 2). En total, los neveros de las Joyas del Nevadín y Valdeiglesia mantienen nieve más de 219 días al año de media, umbral que señalan Andrés de Pablo y Palacios Estremera (2004) para considerar a los nichos activos en la Sierra de Guadarrama.

En los últimos años, salvo los años 2008 y 2009, que han estado más acordes con las precipitaciones medias de nieve para el Alto Sil, el volumen de nieve caída ha sido escaso, lo que se ha traducido en que han sido muy pocas las manchas de nieve que han podido persistir hasta comienzos del verano (Tabla 3). Así, el contraste entre estos años y los anteriores es muy grande, puesto que sólo entre 0 y 5 enclaves llegaron con nieve a comienzos del verano entre 2003 y 2007, mientras que fueron 46 manchas en 2008 y 54 en 2009, lo que indica una fuerte variabilidad inter-anual, dependiente de las condiciones meteorológicas de cada año. En cualquier caso, las condiciones que han permitido la conservación de la nieve han sido diferentes ya que, mientras en 2008 fue la 


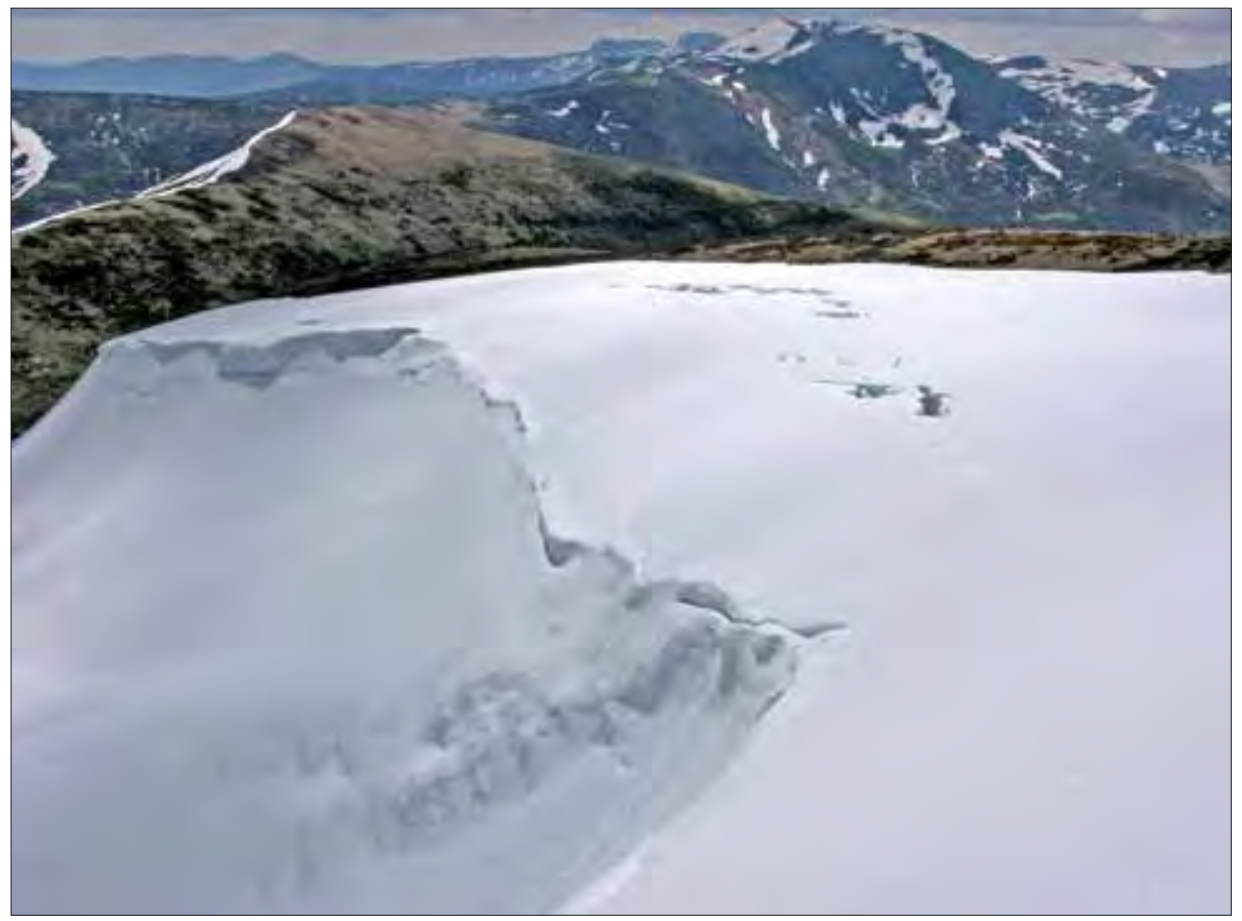

Figura 4. Grietas de tracción y huellas de deslizamientos lentos de nieve en el nevero del Nevadín. Las marcas indican que existen desplazamientos de nieve ladera abajo. El espesor de nieve en ese momento debía superar los 8 metros en algunos sectores del nicho. Abril de 2006.

Tabla 2. Fechas de fusión total y número de días con nieve en los neveros de las Joyas del Nevadin y de Valdeiglesia. * Las fechas indicadas se refieren a la fusión total de la nieve $y$ deben ser tomadas como valor aproximado, con una precisión de \pm 5 días.

\begin{tabular}{|c|c|c|c|c|}
\hline Años & \multicolumn{2}{|c|}{ Joyas del Nevadín* } & \multicolumn{2}{c|}{ Valdeiglesia* } \\
\hline & Fecha Fusión & Días con nieve & Fecha Fusión & Días con nieve \\
\hline $2002-2003$ & 22 de Julio & 251 & 27 de Junio & 225 \\
\hline $2003-2004$ & 25 de Junio & 246 & 10 de Junio & 226 \\
\hline $2004-2005$ & 5 de Junio & 203 & 27 de Mayo & 192 \\
\hline $2005-2006$ & 19 de Julio & 257 & 24 de Junio & 231 \\
\hline $2006-2007$ & 19 de Junio & 216 & 2 de Junio & 197 \\
\hline $2007-2008$ & 10 de Julio & 233 & 1 de Julio & 222 \\
\hline $2008-2009$ & 9 de Julio & 256 & 29 de Junio & 244 \\
\hline Media & 3 de Julio & 237 & 17 de Junio & 220 \\
\hline
\end{tabular}


Tabla 3. Neveros con nieve a comienzo del verano (21 de junio) entre 2003 y 2009. Los números entre paréntesis indican el número de neveros en un mismo entorno.

\begin{tabular}{|c|c|c|c|c|c|c|c|}
\hline Año 2003 & & Año 2004 & \multicolumn{2}{|c|}{ Año 2005} & \multicolumn{2}{|c|}{ Año 2006} & Año 2007 \\
\hline Joyas del Nevadín & \multicolumn{2}{|c|}{ Joyas del Nevadín } & & & \multicolumn{2}{|c|}{ Joyas del Nevadín } & \\
\hline Valdeiglesia & \multicolumn{2}{|r|}{ Los Fueyos } & & & \multicolumn{2}{|c|}{ Valdeiglesia } & \\
\hline Los Fueyos & & & & & \multicolumn{2}{|c|}{ Nevadín Norte } & \\
\hline Peña Carnicera & & & & & \multicolumn{2}{|c|}{ Peña Grande } & \\
\hline \multicolumn{8}{|l|}{ Reguero Joyas } \\
\hline \multicolumn{8}{|c|}{ Año 2008} \\
\hline \multicolumn{2}{|l|}{ Joyas del Nevadín } & \multicolumn{2}{|c|}{ Reguero Joyas } & \multicolumn{2}{|c|}{ Canal Valdeiglesia } & \multicolumn{2}{|c|}{ Peñas del Infierno } \\
\hline \multicolumn{2}{|l|}{ Valdeiglesia } & \multicolumn{2}{|c|}{ Peña Grande } & \multicolumn{2}{|c|}{ Braña la Pena (12) } & \multicolumn{2}{|r|}{ Los Fueyos } \\
\hline \multicolumn{2}{|c|}{ Canchal los Fueyos } & \multicolumn{2}{|c|}{ Joyas Nevadín Sur } & \multicolumn{2}{|c|}{ La Brañina } & \multicolumn{2}{|c|}{ Dos Hermanos Su } \\
\hline \multicolumn{2}{|l|}{ Peña Carnicera (6) } & \multicolumn{2}{|c|}{ Nevadín Norte (3) } & \multicolumn{2}{|c|}{ Braña Librán } & \multicolumn{2}{|c|}{ Circo del Cheiroso } \\
\hline \multicolumn{2}{|c|}{ Circo Peña Carnicera (6) } & \multicolumn{2}{|c|}{ Lóbulos Portilla (2) } & \multicolumn{2}{|c|}{ Ventisquero Portilla (3) } & \multicolumn{2}{|r|}{ Pico la Arena } \\
\hline \multicolumn{8}{|c|}{ Año 2009} \\
\hline \multicolumn{2}{|l|}{ Joyas del Nevadín } & \multicolumn{2}{|c|}{ Reguero Joyas } & \multicolumn{2}{|c|}{ Joyas Nevadín Sur } & \multicolumn{2}{|c|}{ Nevadín Norte (3) } \\
\hline \multicolumn{2}{|l|}{ Circo Nevadín (2) } & Collado N N & & Collado & ista Nevadín & & os Hermanos $\mathrm{N}$ \\
\hline Dos Hermanos $S$ & & Dos Herman & & Valle & el Agua (2) & & Portilla \\
\hline Braña Librán & & Peña Carnic & & & leiglesia & & El Mur (2) \\
\hline Braña la Pena (9 & & Canchal C: & & & Fueyos & & Fasgueo (2) \\
\hline El Miro (6) & & Cueto el $\mathrm{O}$ & & $\mathrm{Bra}$ & a Ronda & & eña Grande (4) \\
\hline
\end{tabular}

existencia de una primavera muy fría la que explicó la persistencia de la nieve, en 2009 fueron las fuertes nevadas a lo largo de todo el invierno las que explican la gran cantidad de nieve que pudo llegar al verano.

En general, al igual que ocurre en la cercana Sierra de Ancares (Pérez Alberti et al., 1998), en los últimos años la persistencia de la nieve en estos enclaves se está reduciendo de manera notable puesto que, según las informaciones recogidas, era habitual que neveros como el de las Joyas del Nevadín, fundieran en el mes de agosto o incluso en septiembre (excepcionalmente todo el año, como ocurrió, probablemente por última vez, en 1992), mientras que actualmente lo suelen hacer a primeros de julio. Si esta dinámica continúa, la presencia de la nieve no será suficiente para mantener las características de un nevero temporal, ya que el ciclo vegetativo no será diferente a otras áreas cercanas y la vegetación podrá desarrollarse normalmente, dificultando los procesos erosivos, como ya ha sucedido en los nichos de nivación situados a menor altitud (Fasgueo y La Bóveda) y está ocurriendo en el resto, aspecto igualmente observado en la Sierra de Guadarrama (Andrés de Pablo y Palacios Estremera, 2004). 


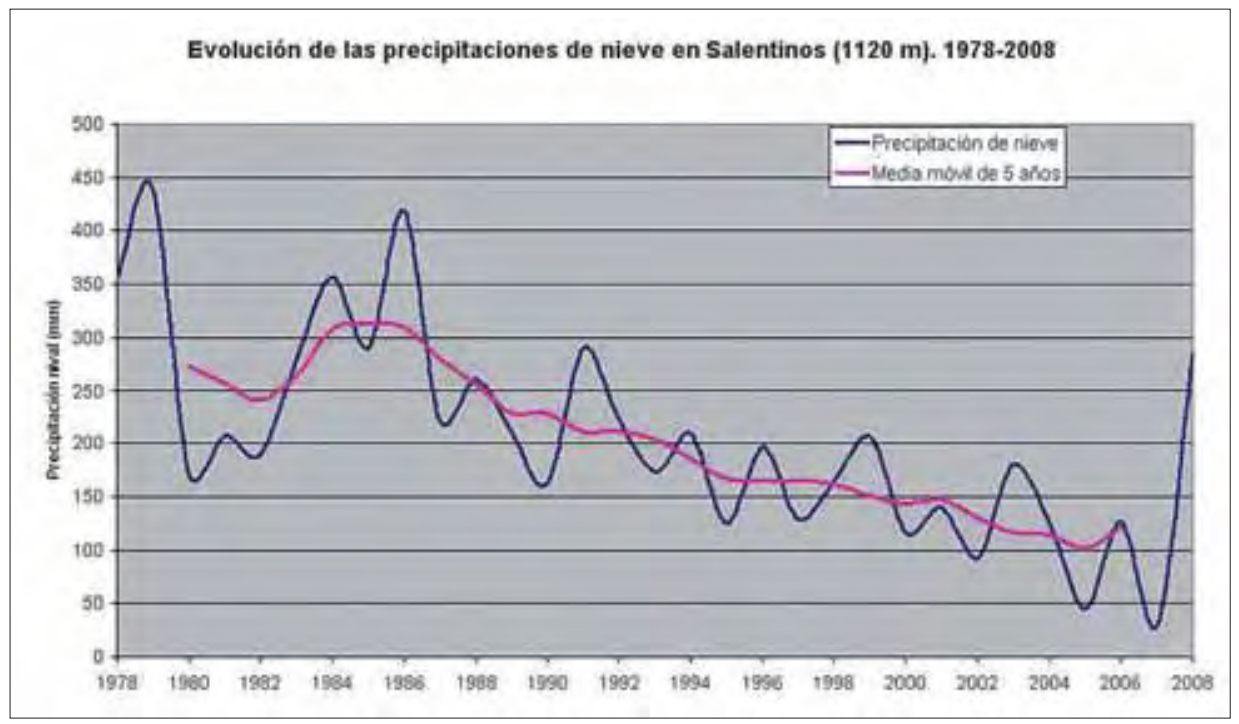

Figura 5. Evolución de las precipitaciones en forma de nieve en Salentinos (1.120 m) entre 1978 y 2008, mostrando una fuerte tendencia regresiva. Destaca el elevado volumen de nieve registrado en 2008, con valores que no se alcanzaban desde 1991. Datos de ENDESA.

La disminución de las precipitaciones en forma de nieve en el Alto Sil es un hecho evidente, al menos desde mediados de los años 80 del pasado siglo (Fig. 5). Esta tendencia regresiva, sólo comprobada en los observatorios, situados siempre en el fondo de los valles, por debajo de los 1.300 metros de altitud, parece ser general a todas las cotas, existiendo una tendencia clara hacia una menor duración del manto nival.

De todos modos, la tendencia regresiva de los nichos de nivación no debe achacarse únicamente a una reducción del volumen de nieve caído, puesto que es muy difícil disociarla de la fuerte reducción de la cabaña ganadera en estas montañas, que ha implicado una mayor colonización de la vegetación arbustiva en cotas altas, reduciendo así el volumen de nieve movilizado por el viento y transportado hacia los nichos.

De nuevo hay que tener en cuenta que son sistemas muy locales y muy dependientes del transporte de nieve en las zonas de barlovento, con lo que un cambio en su entorno puede favorecer cambios notables en la acumulación de nieve. Los neveros del Nevadín y Peña Grande parecen ser los que menos han sufrido la reducción del pastizal en sus zonas de barlovento, lo que ha podido ayudar en la conservación de su dinámica. En este sentido, tras un gran desbroce realizado en el entorno del Valle del Agua (Vivero), dos neveros se han mantenido durante mucho más tiempo del habitual, llegando a comienzos del verano en el año 2009. El cambio de brezal de porte elevado por pastizal parece haber sido el responsable de la mayor acumulación de nieve en este entorno. 


\subsection{La acción erosiva en los neveros}

Una de las acciones más destacables dentro de los nichos es la erosión del sustrato. Esta labor erosiva es siempre más intensa sobre materiales poco resistentes, como se ha comprobado en Groenlandia (Christiansen, 1998) al igual que ocurre con cualquier otro agente erosivo.

Así, en los nichos de nivación del Alto Sil, especialmente en las Joyas del Nevadín, Nevadín Norte y Valdeiglesia existen pequeños afloramientos de rocas que, como hemos podido observar, están siendo erosionados actualmente (Fig. 6), constituyendo una fuente de derrubios para ser removilizados después por la nieve y el agua de fusión. La frescura de muchas de las roturas contrasta con lo que sucede en otros sectores situados a igual cota, donde la estabilidad del roquedo es la nota predominante.

Aunque en principio las numerosas fracturas podrían derivarse de ciclos de hielo/ deshielo, lo cierto es que los registros térmicos obtenidos a nivel superficial dentro del nevero de las Joyas del Nevadín indican que éstos son escasos y se deben a contrastes térmicos poco acusados (Santos González et al., 2009), como ya se observó en otros neveros persistentes (Nyberg, 1991), lo que pone en cuestión la relación entre neveros y la acción de hielo/deshielo (Thorn y Hall, 2002). Por ello, como ya señalara Kariya (2002), quizás sean los ciclos de humectación/secado bajo la nieve los responsables, en mayor medida, de la erosión del sustrato, si bien es un aspecto que deberá ser analizado en el futuro, al igual que la profundidad a la que puede penetrar el hielo dentro de las fisuras de las rocas bajo los neveros, que también podrían explicar la intensa erosión dentro de los nichos de nivación.

En cualquier caso, lo cierto es que la erosión ha sido notablemente más intensa los años con mayor acumulación y persistencia de la nieve; en efecto, mientras años como 2004 y 2005 en los cuales apenas se podían observar pequeñas fracturas aisladas, en 2006 y 2009, tras el período de fusión, eran muy numerosas las fracturas recientes y no sólo se circunscribían a los neveros de las Joyas del Nevadín y Valdeiglesia, sino que aparecían en otros nichos de nivación normalmente poco activos.

Una vez que los materiales quedan sueltos, el agua y la nieve se encargan de movilizarlos ladera abajo, como hemos observado. En este sentido, dentro de los nichos de las Joyas del Nevadín y Valdeiglesia, hemos observado algunas estrías de nevero que evidencian importantes presiones subnivales durante los desplazamientos de la nieve (Fig. 7).

El mecanismo de formación de estrías de nevero ya fue observado por Matthes (1911), con estrías de gran tamaño. Estas estrías han sido señaladas también en un nevero de persistencia similar al del Nevadín, en la cercana Sierra de Ancares, junto al pico Cuiña (1998 m), si bien allí se pueden observar formas más complejas y numerosas (Valcárcel Díaz et al., 2005; Carrera Gómez et al., 2006), que no hemos visto en el Alto Sil, seguramente por la presencia en el Cuiña de dos litologías contrastadas (pizarra y cuarcita), aspecto que no ocurre en los nichos analizados. 


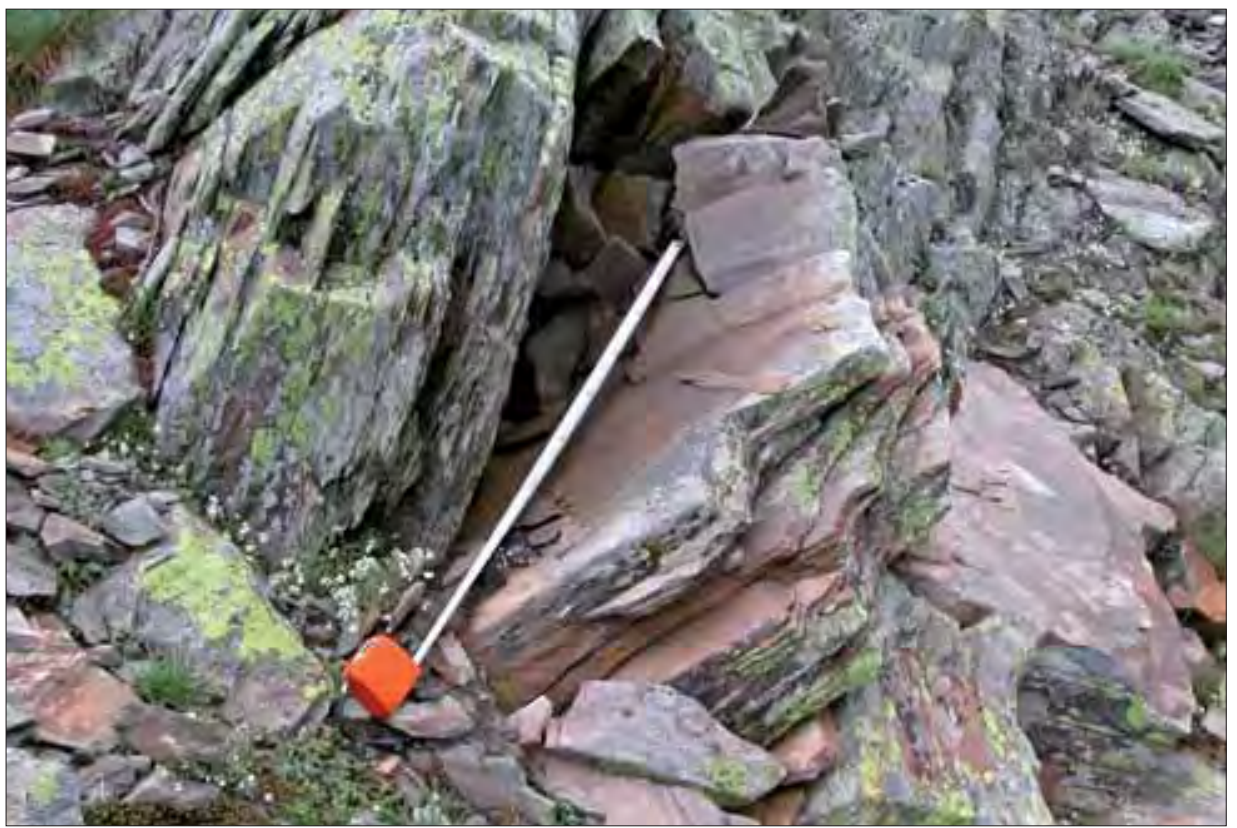

Figura 6. Desalojo por empuje del sustrato de pizarras previamente fracturado en el borde del nevero de las Joyas del Nevadín. La escala es de un metro.

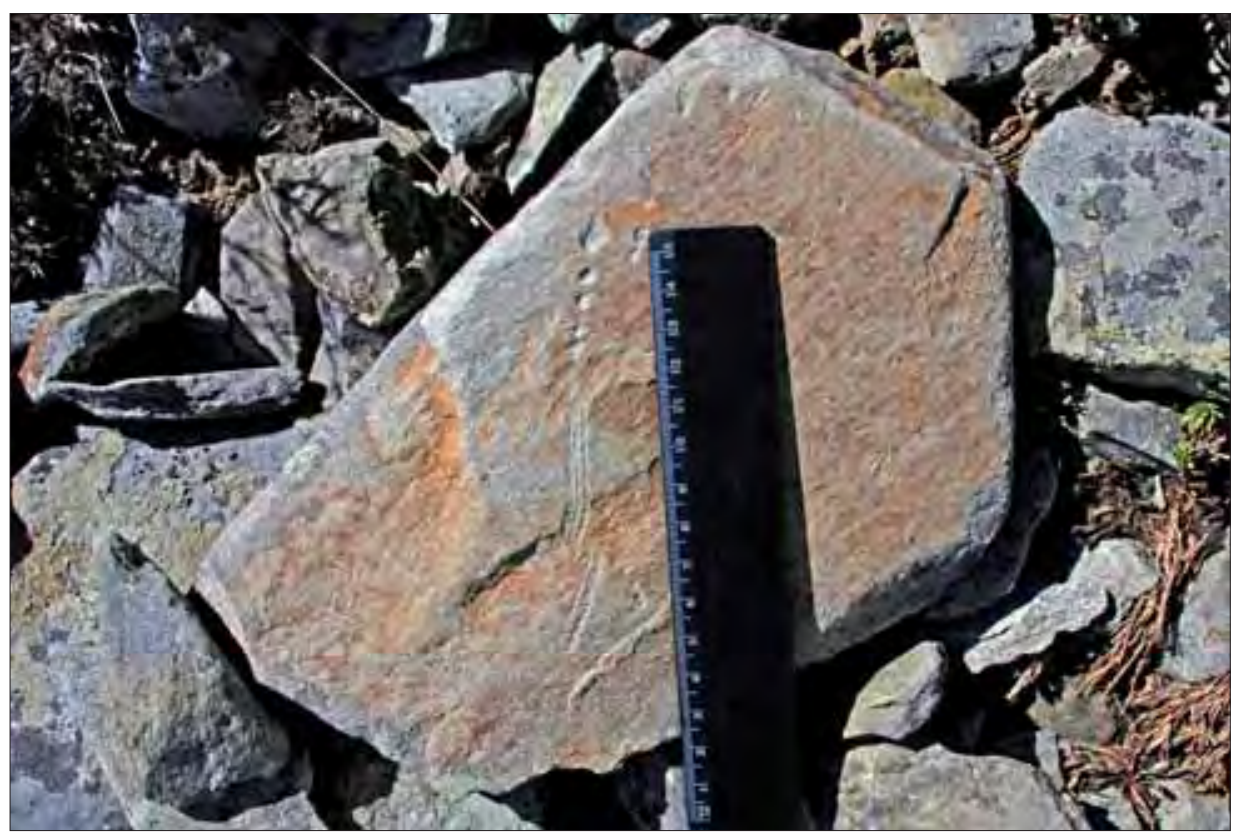

Figura 7. Estrías y marcas de arranque en un canto en el nevero de las Joyas del Nevadín. 


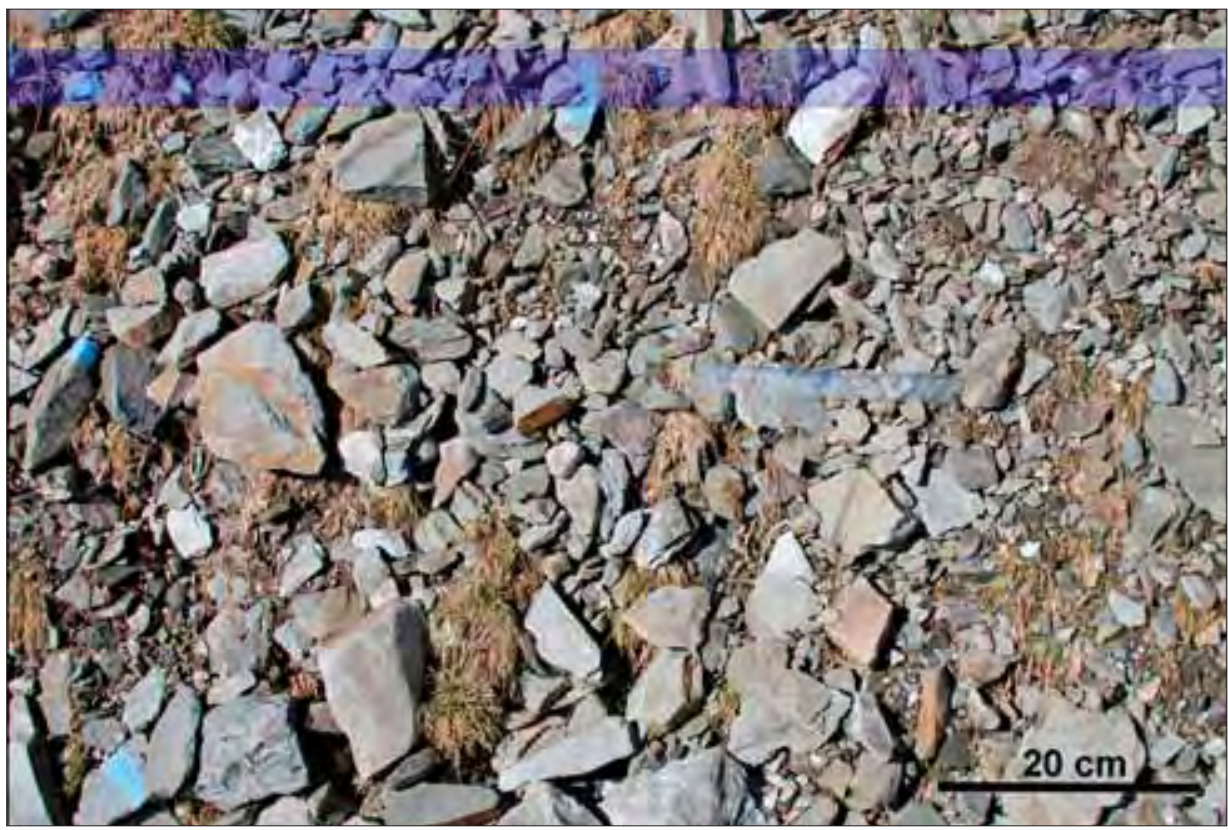

Figura 8. Aspecto de la zona marcada por una línea de pintura en el nevero del Nevadín en junio de 2007, tras menos de cuatro años desde que fue pintada. Se puede apreciar que apenas algún canto está dentro de la zona marcada inicialmente,

dibujada en la fotografía con una franja azul.

En todo caso, su presencia es signo inequívoco de la acción del arrastre de la nieve, creando formas que recuerdan a la acción de un glaciar. Por otra parte, las líneas de pintura trazadas en ambos neveros mostraron evidentes signos de arrastre por parte de la nieve (Fig. 8). Los cantos movilizados, casi en su totalidad, no superan los $5 \mathrm{~cm}$ si bien, ocasionalmente, se han desplazado cantos de hasta $15 \mathrm{~cm}$. En este sentido, se observa una movilidad selectiva de los cantos, puesto que sólo se mueven los más pequeños, mientras que los de más de $25 \mathrm{~cm}$, habitualmente, están cubiertos por líquenes, en consonancia con lo observado en la Sierra de Guadarrama por Palacios Estremera et al. (2002).

Estos arrastres son efectivos en el nevero de las Joyas del Nevadín, donde la gran mayoría de los cantos fueron movilizados, siendo menos frecuentes en el nicho de Valdeiglesia y en el resto de nichos de nivación activos. En cualquier caso, la dinámica es mucho mayor los años en los que los neveros acumulan mayor espesor de nieve, como ocurrió en 2006 y 2009, mientras que es prácticamente nula si no se sobrepasa un umbral de espesor de nieve mínimo. En este sentido, consideramos que sería necesario profundizar en las estimaciones de Bowman (1916) para conocer el umbral de cantidad de nieve acumulada necesaria para que se produzcan desplazamientos de la masa siguiendo la pendiente. 
De todos modos, y aunque la mayor parte de la dinámica se deriva de la acción nival, no debe olvidarse la acción de otros agentes, especialmente del agua, tanto la procedente del deshielo del nevero como de eventos tormentosos de fuerte intensidad. Incluso el viento puede movilizar pequeños cantos que han sido depositados en posiciones inestables por la acción nival.

Por otra parte, con el objetivo de comprobar la capacidad de desplazamiento de la nieve, se instalaron 16 varillas en el nicho de las Joyas del Nevadín y 15 en el de Valdeiglesia, realizándose mediciones de los grados de inclinación de las varillas todos los años tanto al final del período de fusión como en otoño. Todas ellas fueron instaladas con $90^{\circ}$ de inclinación, observándose el doblado de ellas como consecuencia del deslizamiento lento de la nieve (Fig. 9).

La relación del doblado de las varillas con la cantidad y persistencia de la nieve cada año ha sido muy evidente, de tal forma que en años muy escasos en precipitaciones sólidas, como 2005, apenas hubo actividad y quedó restringida al borde superior de

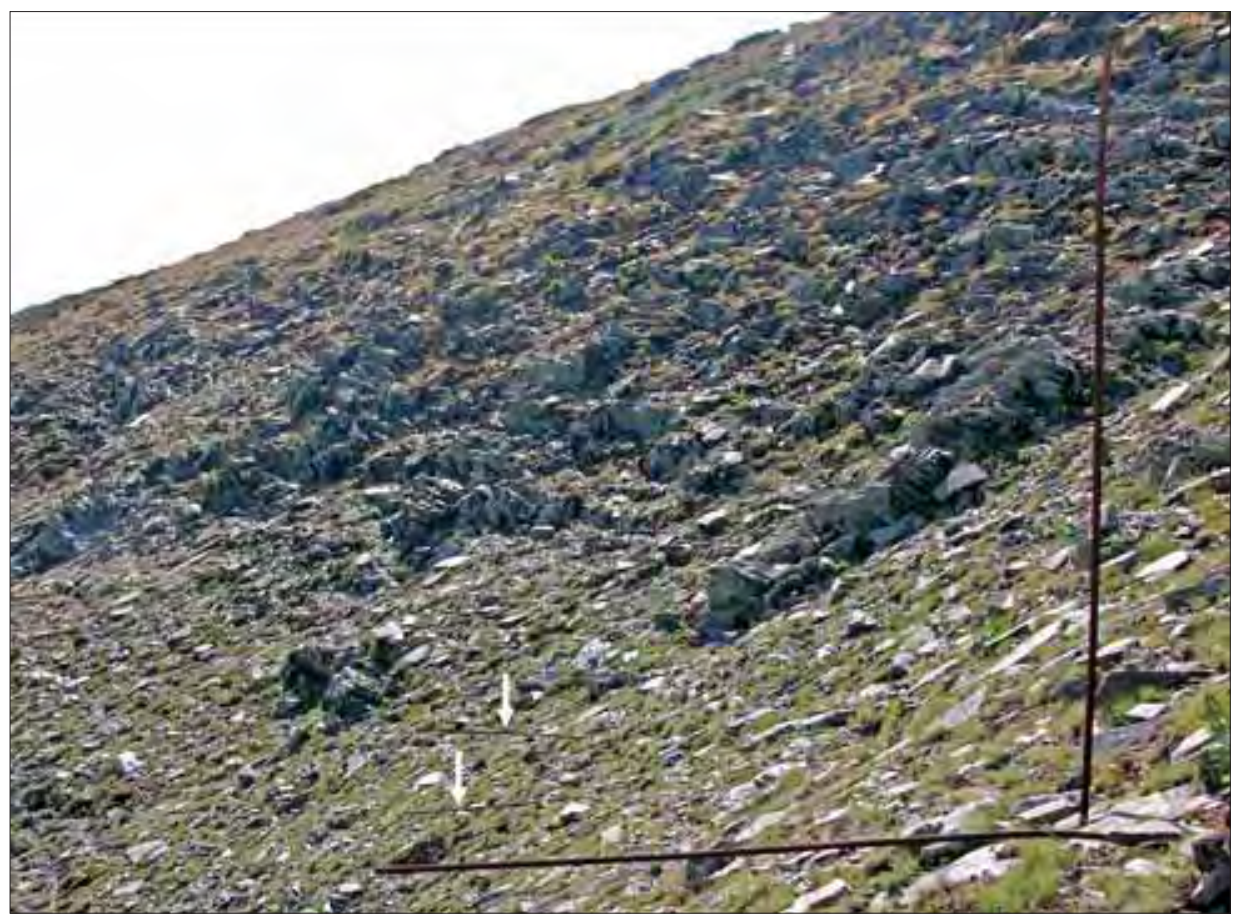

Figura 9. Doblado de varillas de hierro en las Joyas del Nevadín. Se puede observar una varilla prácticamente horizontal por el empuje de la nieve. La varilla vertical acababa de ser instalada para ver la acción nival al invierno siguiente e indica la posición original de la doblada. Al fondo de la imagen son visibles otras dos varillas intensamente dobladas, señaladas por flechas. 
los neveros. Por el contrario, años con gran cantidad de nieve (2006) todas las varillas instaladas quedaron dobladas por efecto del deslizamiento de la nieve (Tabla 4).

Un aspecto que debemos considerar es que las varillas son de hierro, por lo que recuperan parcialmente su posición una vez fundida la nieve, lo que hemos observado realizando mediciones de las varillas parcialmente tapadas por la nieve $\mathrm{y}$, posteriormente, sin nieve. El grado de recuperación ha sido de entre $16^{\circ}$ y $22^{\circ}$, por lo que los valores de la Tabla 4 son inferiores a los reales.

Tabla 4. Porcentaje de varillas de hierro dobladas y grados de inclinación que presentaban en los neveros de las Joyas del Nevadín y Valdeiglesia, en los años 2004, 2005 y 2006.

\begin{tabular}{|c|c|c|c|c|c|c|c|c|c|}
\hline Años & \multicolumn{3}{|c|}{ Año 2004} & \multicolumn{3}{|c|}{ Año 2005} & \multicolumn{3}{|c|}{ Año 2006} \\
\hline \multirow[t]{2}{*}{ Filas } & $\begin{array}{c}\% \text { de } \\
\text { varillas } \\
\text { dobladas }\end{array}$ & $\begin{array}{r}\mathrm{D} \\
\mathrm{m} \\
\text { má }\end{array}$ & $\begin{array}{l}\text { ado } \\
\text { o y } \\
\text { o }\left(^{\circ}\right)\end{array}$ & $\begin{array}{c}\% \text { de } \\
\text { varillas } \\
\text { dobladas }\end{array}$ & & $\begin{array}{l}\text { ado } \\
\text { o y } \\
\text { lo }\left({ }^{\circ}\right)\end{array}$ & $\begin{array}{c}\% \text { de } \\
\text { varillas } \\
\text { dobladas }\end{array}$ & $\begin{array}{r}\text { Do } \\
\text { me } \\
\text { máx }\end{array}$ & $\begin{array}{l}\text { lado } \\
\text { io y } \\
\text { no }\left(^{\circ}\right)\end{array}$ \\
\hline & \multicolumn{3}{|c|}{ Nevadín } & \multicolumn{3}{|c|}{ Nevadín } & \multicolumn{3}{|c|}{ Nevadín } \\
\hline Fila superior & 66 & 29 & 44 & 75 & 17 & 28 & 100 & 70 & 119 \\
\hline Fila media & 28 & 5 & 18 & 28 & 3 & 15 & 100 & 63 & 104 \\
\hline Fila inferior & 0 & 0 & 0 & 0 & 0 & 0 & 100 & 39 & 50 \\
\hline \multirow[t]{2}{*}{ Total } & 25 & 8 & 44 & 29 & 5 & 28 & 100 & 58 & 119 \\
\hline & \multicolumn{3}{|c|}{ Valdeiglesia } & \multicolumn{3}{|c|}{ Valdeiglesia } & \multicolumn{3}{|c|}{ Valdeiglesia } \\
\hline Fila superior & 33 & 4 & 15 & 0 & 0 & 0 & 100 & 71 & 78 \\
\hline Fila media & 0 & 0 & 0 & 0 & 0 & 0 & 100 & 49 & 54 \\
\hline Fila inferior & 17 & 2 & 9 & 0 & 0 & 0 & 100 & 33 & 38 \\
\hline Total & 19 & 2 & 15 & 0 & 0 & 0 & 100 & 51 & 78 \\
\hline
\end{tabular}

\section{Conclusiones}

Las observaciones realizadas entre 2003 y 2009 demuestran que existe una dinámica nival activa en algunos de los nichos de nivación del Alto Sil, en especial en las Joyas del Nevadín y, en menor medida, en Valdeiglesia. El desgaste de los afloramientos rocosos bajo los neveros es notable, si bien no se ha podido determinar en qué medida las fracturas observadas son debidas a ciclos de hielo/deshielo o a ciclos de humectación/secado.

Los deslizamientos lentos de la nieve han sido probados gracias a la observación del doblado de varillas de hierro en los neveros de las Joyas del Nevadín y de Valdeiglesia, así como a la movilidad de cantos y la presencia de estrías de nevero. Esta dinámica es mucho más intensa los años en los que la cantidad de nieve acumulada es muy grande, siendo casi nula los años en los que espesor de nieve es escaso, como sucedió en 2005. 
Creemos que los procesos erosivos dependen más del espesor y transformación de la nieve dentro del nicho que de la permanencia total de la nieve que, ocasionalmente, puede alargarse debido a ritmos de fusión más bajos de los habituales, como ocurrió en 2008. De todos modos, y puesto que el espesor de nieve que se alcanza y su duración están muy relacionados, a falta de observaciones más detalladas, el umbral de 219 días de nieve (Andrés de Pablo y Palacios Estremera, 2004) parece adecuado para separar los nichos de nivación activos de los inactivos, teniendo siempre presente la fuerte variabilidad interanual existente.

En los últimos años parece existir una notable disminución del volumen de nieve que reciben estas montañas, lo que se traduce en una dinámica menos activa (o activa menos años) de los nichos de nivación y, por consiguiente, en una progresiva colonización de la vegetación, que estabiliza estos enclaves. De todos modos, el cambio de usos del suelo tiene igualmente una importancia significativa en la acumulación de nieve, lo que hace necesario profundizar en las relaciones entre la dinámica geomorfológica, la vegetación, los usos del suelo y el clima.

\section{Agradecimientos}

Este trabajo ha sido posible gracias a una beca doctoral de la Universidad de León para la realización del trabajo titulado "Glaciarismo y periglaciarismo en el Alto Sil, provincia de León (Cordillera Cantábrica)”. Así mismo, se enmarca dentro del proyecto "Dinámica geomorfológica en el entorno de los glaciares rocosos de la Cordillera Cantábrica”, (LE020A07), financiado por la Junta de Castilla y León.

Nuestro agradecimiento a diversas personas por la información aportada acerca de la persistencia de la nieve en diversos enclaves, en especial a Vitín y Saúl, de Salientes.

\section{Referencias bibliográficas}

Andrés de Pablo, N., PAlacios Estremera, D. (2004). Interrelación nieve/geomorfología en la Sierra de Guadarrama: altas cuencas del Ventisquero de La Condesa y Valdemartín. Cuadernos de Investigación Geográfica, 30: 85-116.

Bowman, I. (1916). The Andes of southern Peru. Geographical Reconnaisance Along the Seventy-Third Meridian. American Geographical Society, Special Publication: 295 pp, New York.

Carrera Gómez, P., Valcárcel Díaz, M., Pérez Alberti, A. (2006). Ejemplos de formas erosivas de origen nival en las vertientes del alto Ancares, noroeste de la provincia de León. En: Geomorfología y territorio. Actas de la IX Reunión Nacional de Geomorfología (Pérez Alberti, A., López Bedoya, J., Eds.). Universidade de Santiago de Compostela, pp. 59-65, Santiago de Compostela. 
Christiansen, H. H. (1998). Nivation forms and processes in unconsolidated sediments, NE Greenland. Earth Surface Processes and Landforms, 23: 751-760.

Corripio, J. G., Durand, Y., Guyomarc'H, G., Merindol, L., Lecorps, D., Pugliese, P. (2004). Land-based remote sensing of snow for the validation of a snow transport model. Cold Regions Science and Technology, 39: 93-104.

Costin, A. B., Jennings, J. N., Bautovich, B. C., Winbush, D. J. (1973). Forces developed by snowpatch action, Mt. Twynam, Snowy Mountains, Australia. Arctic and Alpine Research, 5: 121-126.

Daimaru, H., Ohtani, Y., Ikeda, S., OKamoto, T., Kajimoto, T. (2002). Paleoclimatic implication of buried peat layers in a subalpine snowpatch grassland on Mt. Zarumori, northern Japan. Catena, 48: 53-65.

DAvid, E. (2004). "Get the drift" effects of snow fence variables on wind patterns, snow drift geometry and volume. ISSW 2004 Preceedings. A merging of theory and practice. International Snow Science Workshop, pp. 217-226, Jackson Hole (Wyoming).

Evans, I. S., Cox, N. J. (1974). Geomorphometry and the operational definition of cirques. Area, 6: 150-153.

Fernández Martínez, E., Fuertes GutiérRez, I. (Coords.) (2009). Lugares de Interés Geológico. León. Junta de Castilla y León, DVD publicado por la Fundación Patrimonio Natural.

García de Celis, A., Martínez Fernández, L. C. (2002). Morfología glaciar de las montañas de la cuenca alta de los ríos Sil, Omaña, Luna y Bernesga: revisión y nuevos datos (Montaña Occidental de León). En: El modelado de origen glaciar en las montañas leonesas (Redondo, J.M., Gómez, A., González Gutiérrez, R.B., Carrera Gómez, P., Coords.). Universidad de León, pp. 137-196, León.

GARDNER, J. (1969). Snowpatches: their influence on mountain wall temperatures and geomorphic implications. Geografiska Annaler, 51 A (3): 114-120.

GonzÁlez GutiérRez, R. B. (2002). El relieve de los valles del Torío y Curureño (Montaña Cantábrica Leonesa). Universidad de León, 267 pp., León.

Guyomarc'h, G., Durand, Y., Lecorps, D., Mérindol, L., Puglièse, P. (2004). Modeling and validation of snow redistribution by wind. ISSW 2004 Preceedings. A merging of theory and practice. International Snow Science Workshop, pp. 820828, Jackson Hole (Wyoming).

Jalut, G., Belet, J. M., García de Celis, A., Redondo Vega, J. M., Bonnet, L., Valero Garcés, B., Moreno, A., Villar Pérez, L., Fontugne, M., Dedoubat, J. J., González Sampériz, P., Santos Fidalgo, L., Vidal Romanì, J. R. (2004). Reconstrucción paleoambiental de los últimos 35000 años en el noroeste de la Península Ibérica: La Laguna de Villaseca (León). Geo-Temas, 6 (5): 105-108. 
Jennings, J. N., Costin, A. B. (1978). Stone movement through snow creep, 1963-75 Mount Twynam, Snowy Mountains, Australia. Earth Surface Processes and Landforms, 3 (1): 3-22.

KariYA, Y. (2002). Geomorphic Processes at a Snowpatch Hollow on Gassan Volcano, Northern Japan. Permafrost and Periglacial Processes, 13: 107-116.

LEwIS, W. V. (1939). Snow-patch erosion in Iceland. The Geographical Journal, 94 (2): 153-161.

Mases, M., Font, D., Vilaplana, J. M. (1998). Relationship between snowdrift development and drifted snow during a wind episode. Annals of Glaciology, 26: 451-455.

Matthes, F. E. (1911). The striped rock floor of the Little Yosemite. Sierra Club Bulletin, 8 (1): 3-9.

Nelson, F. E. N., JACKSON, L. E. (2003). Cirque forms and alpine glaciation during the Pleistocene, west-central Yukon. En: Yukon Exploration and Geology 2002 (Emond, D.S., Lewis, L.L., Eds.). Exploration and Geological Services Division, Yukon Region, Indian and Northern Affairs Canada, pp. 183-198.

NyBERG, R. (1991). Geomorphic processes at snowpatch sites in the Abisko mountains, northern Sweden. Zeitschrift für Geomorphologie N.F., 35 (3): 321-343.

Palacios Estremera, D., Andrés de Pablo, N., Luengo Nicolau, E. (2002). Localización de los procesos actuales en el Circo de Peñalara y su relación con la cubierta nival. En: Aportaciones a la Geomorfología de España en el inicio del Tercer Milenio (Pérez González, A., Vegas, J., Machado, M.J., Eds.). Instituto Geológico y Minero de España, pp. 431-440, Madrid.

Palacios, D., Andrés, N., Luengo, E. (2003). Distribution and effectiveness of nivation in Mediterranean mountains: Peñalara (Spain). Geomorphology, 54: 157-178.

Palacios Estremera, D., Andrés de Pablo, N., Luengo Nicolau, E. (2004). Tipología y evolución de los nichos de nivación en la Sierra de Guadarrama, España. Boletín de la Real Sociedad Española de Historia Natural, 99 (1-4): 141-158.

Palacios, D., García SÁnchez-Colomer, M. (1997). The influence of geomorphologic heritage on present nival erosion: Peñalara, Spain. Geografisca Annaler, 79 A (1-2): 25-40.

Pérez Alberti, A., Valcárcel díaz, M., Martínez Cortizas, A., Blanco Chao, R. (1998). Evidencias de procesos periglaciares actuales en el noroeste de la Península Ibérica. En: Procesos biofísicos actuales en medios fríos (Gómez Ortiz, A., Salvador Franch, F., Schulte, L., García Navarro, A., Eds.). Publicacions de la Universitat de Barcelona, pp. 245-261, Barcelona.

Redondo vega, J. M. (2002). El relieve glaciar de la Sierra de Gistredo (NW de la Cordillera Cantábrica, León). En: El modelado de origen glaciar en las montañas leo- 
nesas (Redondo Vega, J.M., Gómez Villar, A., González Gutiérrez, R.B., Carrera Gómez, P., Coords.). Universidad de León, pp. 105-136, León.

Redondo vega, J. M., Gómez villar, A., González Gutiérrez, R. B. (2004). Localización y caracterización morfométrica de los glaciares rocosos relictos de la Sierra de Gistredo (Montaña Cantábrica, León). Cuadernos de Investigación Geográfica, 30: $35-60$.

Redondo Vega, J. M., GonZÁlez Gutiérrez, R. B., SANTOS GOnZÁlez, J., GómeZ ViLlar, A. (2006). Sedimentación glaciolacustre en la Montaña Cantábrica Leonesa, pp. 83100. En: Geomorfología y territorio (Pérez Alberti, A., López Bedoya, J., Eds.). Universidade de Santiago de Campostela: pp. 83-100, Santiago de Compostela.

Santos gonzÁlez, J., Redondo vega, J. M., Gómez villar, A., González Gutiérrez, R. B. (2006). Bloques erráticos en Páramo del Sil: testigos del máximo avance glaciar en la cuenca del Sil (Cordillera Cantábrica). En: Geomorfología y territorio (Pérez Alberti, A., López Bedoya, J., Eds.). Universidade de Santiago de Campostela, pp. 101-112, Santiago de Compostela.

Santos gonzÁlez, J., GonzÁlez Gutiérrez, R. B., Gómez villar, A., Redondo vega, J. M. (2009). Ground thermal regime in the vicinity of relict rock glaciers (Cantabrian Mountains, NW Spain). Finisterra, 87: 35-44.

Serrano, E., Martínez de Pisón, E., Agudo, C. (2000). El medio periglaciar de alta montaña en el Pirineo central: aportaciones recientes. En: Procesos y formas periglaciares en la alta montaña mediterránea (Peña Monné, J.L., Sanchez Fabre, M., Lozano Tena, M.V., Eds.). Instituto de Estudios Turolenses, pp. 45-62, Teruel.

Shakesby, R. A. (1997). Pronival (protalus) ramparts: a review of forms, processes, diagnostic criteria and palaeoenvironmental implications. Progress in Physical Geography, 21 (3): 394-418.

Shakesby, R. A., Matthews, J. A., McEwen, L. J., Berrisford, M. S. (1999). SnowPush Processes in Pronival (Protalus) Rampart Formation: Geomorphological Evidence from Smørbotn, Romsdalsalpane, Southern Norway. Geografiska Annaler, 81 (1): $31-45$.

Thorn, C. E., Hall, K. (2002). Nivation and cryoplanation: the case for scrutiny and integration. Progress in Physical Geography, 26 (4): 533-550.

Valcárcel díaz, M., Carrera Gómez, P., Pérez Alberti, A. (2005). Nival bedrock erosion at a seasonal snow patch site from the Cuiña cirque, Ancares Sierra, northwestern Spain. En: Shifting lands, new insights into periglacial geomorphology (Etienne, S., Ed.). ESF-Sediflux Network, second conference, Clermont-Ferrand, Coll. Géoenvironnement, Sétun, pp. 94-95, Clermont-Ferrand.

VAN Everdingen, R. O. (2005). Multi-language glossary of permafrost and related ground-ice terms. International Permafrost Association. The Arctic Institute of North America, The University of Calgary, Calgary.

Watson, A., Davison, R. W., French, D. D. (1994). Summer snow patches and climate in north-east Scotland. Arctic and Alpine Research, 26 (2): 141-151. 